\title{
Basins of Attraction and Perturbed Numerical Solutions using Euler's Method
}

\author{
Hendrik Orem \\ Harvey Mudd College \\ hendrik_orem@hmc.edu \\ Sponsor: Professor Rachel Levy \\ Harvey Mudd College \\ levy@hmc.edu
}

\begin{abstract}
Small uncertainties in a dynamical system due to imperfect measurements or variations in the environment can dramatically impact the long term behavior of a trajectory. This phenomenon is studied in a population competition model by introducing a random error term into a numerical solver and investigating the effect on the behavior of solutions. Two methods for analyzing the impact of a random term are demonstrated.
\end{abstract}

\section{Introduction}

\subsection{Motivation}

Any physical system corresponding to a two-dimensional vector field has some uncertainty in the true flow at any given point; this uncertainty may even vary in time. Analytic study of a dynamical system often shows that a given point is in the basin of attraction of an asymptotically stable fixed point, but uncertainty in the vector field may change the fixed point to which a trajectory is attracted. In many applications of dynamical systems, it is important to understand the impact of unexpected variations in the vector field.

This paper investigates a particular two-dimensional vector field with numerical methods by perturbing the solution at each iteration of Euler's method. We take the perturbation to be uniformly distributed in some interval $[-k, k]$, and study the impact of varying the parameter $k$ on the probability of a given initial condition tending towards one fixed point or the other.

\subsection{Basic Problem}

We will consider the system

$$
\left[\begin{array}{c}
\dot{x} \\
\dot{y}
\end{array}\right]=\left[\begin{array}{c}
x(3-x)-2 x y \\
y(2-y)-x y
\end{array}\right]
$$

which models the competition between populations of rabbits and sheep as described by Strogatz [3], an excellent undergraduate text in dynamical systems. The first term in each 


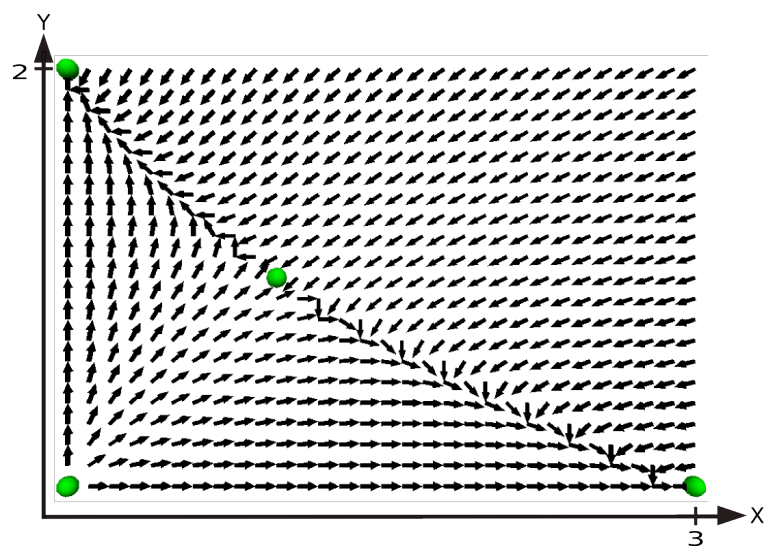

Figure 1: The vector field in system (1).

component represents population growth up to the environment's carrying capacity (i.e. the logistic equation). The coupling term in each component encodes the fact that the two species eat the same food source and hence each has a negative impact on the other's growth. The system has four fixed points, namely

(a) $(0,0)$, an unstable fixed point;

(b) $(3,0)$, a stable fixed point;

(c) $(0,2)$, a stable fixed point;

(d) $(1,1)$, a saddle point.

The fixed points are shown as circles on the plot in Figure 1; arrows indicate the direction of the vector field. Most points are attracted to either $(3,0)$ or $(0,2)$, the two asymptotically stable fixed points. The boundary of the basin of attraction, the stable manifold of $(1,1)$, can be approximated using the linearization of the system at the saddle point $(1,1)$. In particular, the eigenvector $\left[\begin{array}{c}\sqrt{2} \\ 1\end{array}\right]$ along the fixed point's stable manifold locally defines the boundary between the two regions.

Consider the process of perturbing a numerical solution to this system starting from a point $\left(x_{0}, y_{0}\right)$. It may be that the trajectory starting at $\left(x_{0}, y_{0}\right)$ is 'pushed' into the opposite basin of attraction so that it ends at the opposite fixed point from the one it would have tended towards in a nonperturbed solution. Studying the proportion of randomly perturbed numerical solutions with initial point $\left(x_{0}, y_{0}\right)$ that are attracted to each fixed point can provide some measure of the 'strength' of a basin of attraction. 


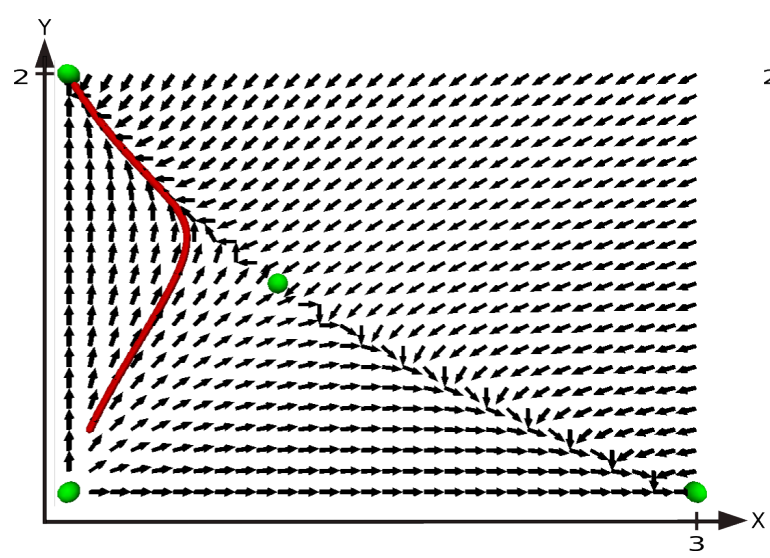

(a) Unperturbed Trajectory

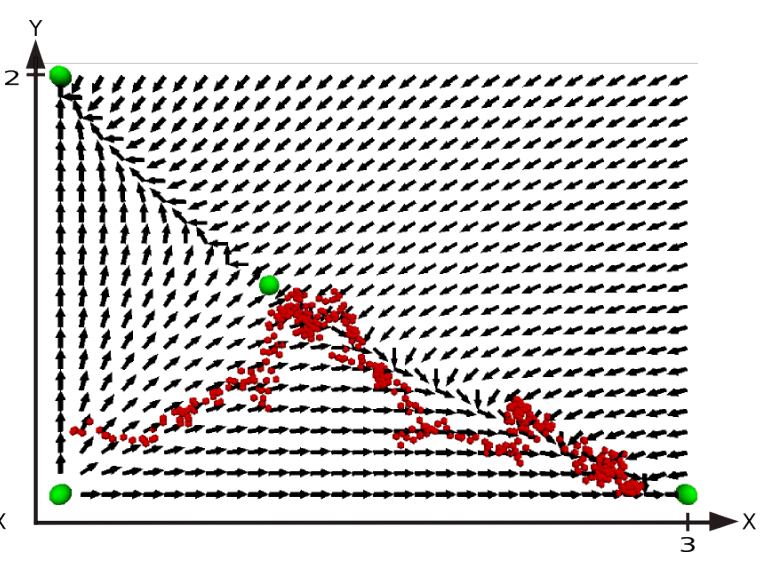

(b) Perturbed Trajectory, $k=0.1$

Figure 2: Two trajectories starting from $(0.1,0.3)$. The perturbed trajectory ends at the fixed point $(3,0)$, even though it is in the basin of attraction of $(0,2)$, as seen in the unperturbed trajectory.

\section{Methodology}

\subsection{Formulation: Euler's Method}

Starting from a point $\vec{x}_{0}$, we compute the nonperturbed trajectory for the system $\dot{\vec{x}}=f(\vec{x})$ using the explicit form of Euler's method:

$$
\vec{x}_{n+1}=\vec{x}_{n}+h \dot{\vec{x}}_{n}
$$

where $h$ is the step size. In the perturbed case there is an addtional term:

$$
\left.\vec{x}_{n+1}=\vec{x}_{n}+h \dot{\vec{x}}_{n}+k \text { (uniform }[-1,1]\right),
$$

where uniform $[-1,1]$ chooses a random real number that is uniformly distributed in $[-1,1]$ and the scalar $k$ tells us the magnitude of the perturbation.

Applying the Euler method in (2) to system (1) with $\left(x_{0}, y_{0}\right)=(0.1,0.3)$ gives the plot shown in Figure 2a, where the trajectory is indicated with a solid line. We see that it is in the basin of attraction of $(0,2)$. When we instead apply the perturbed solver in (3) with $k=0.1$, the trajectory approaches the fixed point at $(3,0)$ as seen in Figure 2b.

To understand the impact of the perturbation term, the following section describes the frequency of such reversals of fixed point attraction for initial points $\left(x_{0}, y_{0}\right)$ in the first quadrant of the vector field in terms of the magnitude of the perturbation, namely the parameter $k$ in equation (3). Unless otherwise stated, all figures were generated by discretizing the first quadrant with increments of size 0.1 subject to $x \leq 3$ and $y \leq 2$ with $k=0.05$.

All figures were generated in the Python programming language using either the VPython module [4] or Matplotlib [1]. Random numbers used in method (3) were computed using the standard Python random module, which creates pseudo-random numbers using an algorithm suitable for most non-cryptographic purposes [2]. 


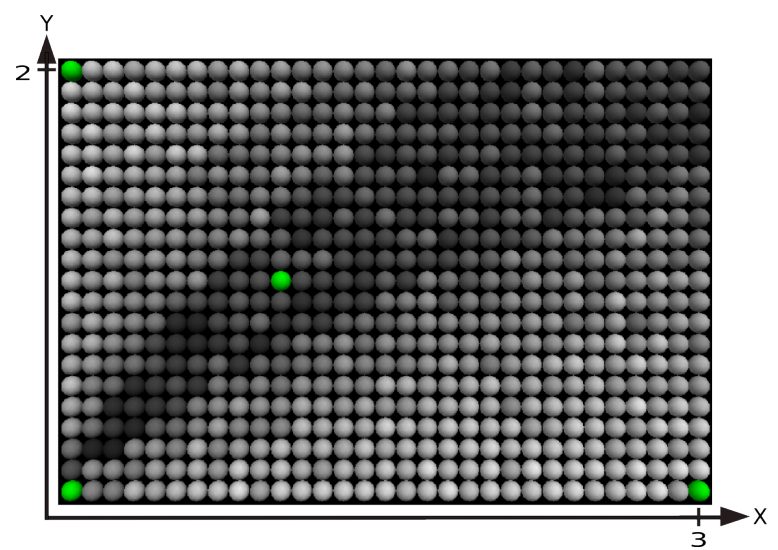

Figure 3: Stability of the plane with a perturbation magnitude $k=0.05$; stability 1 is white, stability 0 is black. Notice that there is a region of low stability along the eigenvector of the fixed point $(1,1)$ corresponding to the boundary of the basin of attraction.

\subsection{Stability Metric}

We will study the effect of perturbations on basins of attraction using a notion of average stability of initial points, starting at each point of an evenly spaced grid in the first quadrant (the region where system (1) is defined). We define the stability of a point $\left(x_{0}, y_{0}\right)$ to be the fraction of perturbed trajectories starting at $\left(x_{0}, y_{0}\right)$ which are attracted to the same fixed point under the perturbed method in (3) as in the normal Euler solver in (2). Thus a stability value of 1 indicates that all perturbed trajectories go to the same fixed point as the normal trajectory, 0.5 indicates that half do, and so on.

Computing stability analytically is prohibitively complicated, so we approximate it by comparing the results of $N$ perturbed solutions starting from $\left(x_{0}, y_{0}\right)$ running for $S$ steps with the result of a nonperturbed solution. A given fixed point is said to be the 'result' of a solution if, after $S$ steps, it is the nearest fixed point and the solution is within a distance of $10^{4}$ of the origin (to exclude pathological solutions which were pushed out of the first quadrant).

\section{Results}

\subsection{Graphical Study of Uniform Perturbation}

We offer two visual ways to study how the stability of points, defined in Section 2.2, decreases as the distance from attracting fixed points increases. First, we can gain a qualitative sense of the impact of the distance from a fixed point on stability by examining Figure 3. At each discretized point, $N=30$ trials were run for $S=300$ steps with a step size of 0.01 to estimate the stability. A stability of 1 is indicated with a white circle, stability 0 with a black circle, and stabilities inbetween are shaded linearly in grayscale.

The behavior is roughly as expected in that the stability is highest close to the stable 


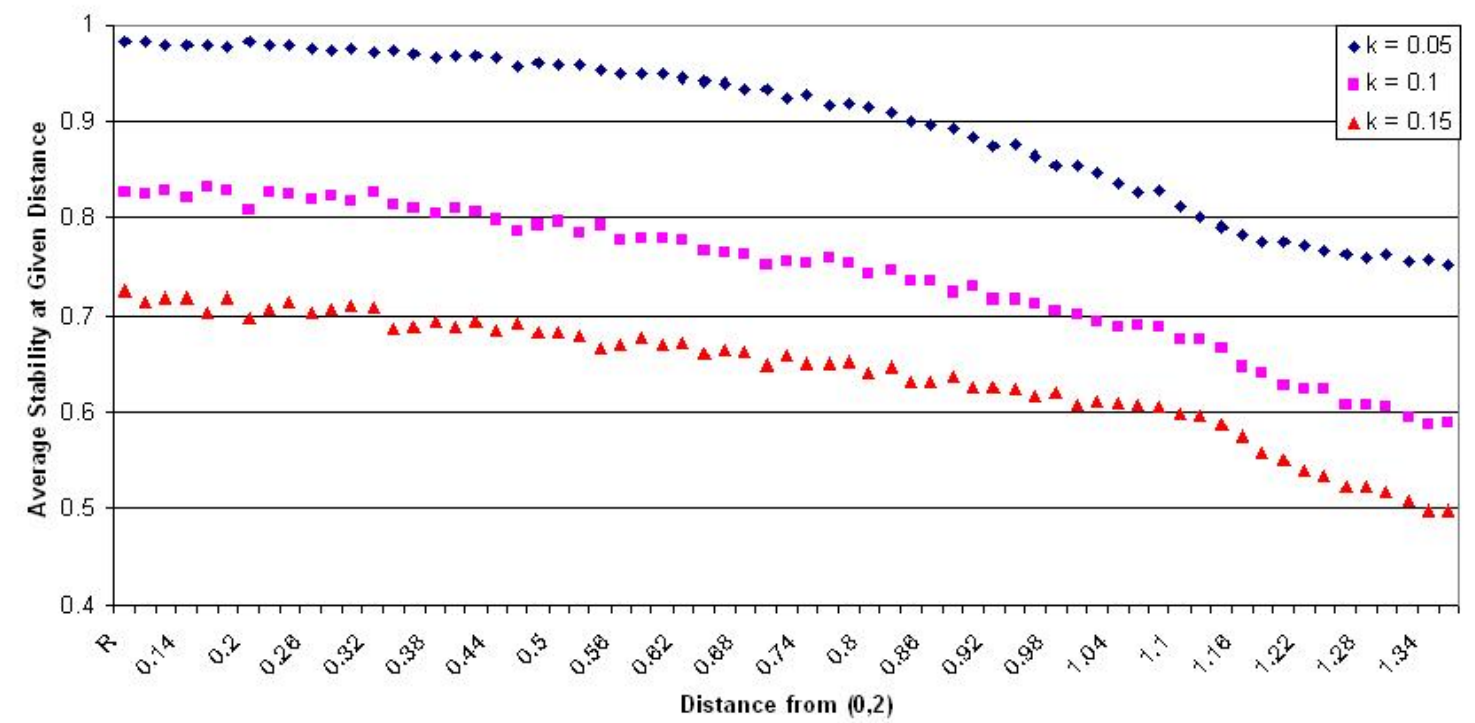

Figure 4: Plot of average stability at radius $R$ versus radius for three values of perturbation magnitude about the fixed point $(0,2)$ with $N=30$ and $S=300$. Notice that each increase of $k$ by 0.05 shifts the distribution down while keeping the shape roughly constant, suggesting a simple dependence on $k$.

fixed points and decreases to zero close to the line defined by the eigenvector $\left[\begin{array}{c}\sqrt{2} \\ 1\end{array}\right]$ of the saddle point at $(1,1)$. This is expected because the eigenvector is roughly the border of the basin of attraction. The darkened region in Figure 3 becomes wider as $x$ and $y$ increase. Near the origin, the unstable fixed point at $(0,0)$ appears to have the effect of increasing stability by 'pushing' trajectories away into their respective basins of attraction.

\subsection{Average Stability Distribution}

Figure 4 provides a more quantitative image of how stability varies away from the fixed point $(0,2)$. The horizontal axis represents distance from the fixed point, $R$. The vertical axis displays the average stability of all points in the discretized first quadrant in the area between the circles of radius $R+g$ and $R-g$ centered at the fixed point; $g$ is the gradation used to discretize the plane, which was reduced to 0.02 in this computation to improve resolution.

Comparing the three data sets shown in Figure 4 provides rudimentary insights into the impact of the parameter $k$ in (3) on the stability distribution about $(0,2)$. All three distributions display roughly the same shape as they drop off from a maximum value at small radius. The lowest shows a less smooth shape, as expected given the larger perturbation.

In spite of the increased variability of the data points in the lower two data sets, there is a clear impact of increasing $k$ on the distribution of stabilities about $(0,2)$. The shape of 
all three distributions is roughly the same, but the first varies from an upper bound near 1 to a lower bound near 0.75 ; the second also has a range of 0.25 but shifted down by 0.15. The final graph is also shifted down, but the points are too variable to determine the magnitude of the shift; the data are consistent with a downward shift of 0.10 , and a rerun of the $k=0.15$ case in Figure 4 with 50 rather than 30 runs per point supports a downward shift of 0.10 .

This would suggest that the distribution of stabilities shifts sublinearly with $k$. That is to say, the highest point on the graphs shown in Figure 4, along with the rest of the distribution, changes more slowly as a function of the magnitude of the perturbation than a constant multiple of magnitude would.

\subsection{Implications of Results}

The results indicate that average stability of points a radius $R$ from the fixed point $(0,2)$ varies sublinearly with the constant $k$. Suppose that we physically interpret the magnitude of $k$ to indicate the level of uncertainty in the direction of the flow; this means that the average probability of being 'pushed' into the opposite basin of attraction for a given distance from the fixed point could possibly be predicted from an understanding of the uncertainty in the system.

When studying a dynamical system, we are often concerned with basins of attraction. Small variations in the vector field due to uncertainty in measurement or gradual changes in the physical system could lead to inaccuracies in analytical results; the sort of analysis presented above provides a way of mitigating this concern. Sublinear dependence of stability on uncertainty means that we can, for example, analyze the tradeoff of stability versus the cost of an experiment by determining what level of uncertainty in the result corresponds to what level of detail in measurement.

\section{Conclusion}

\subsection{Summary}

We have considered the dynamical system

$$
\left[\begin{array}{c}
\dot{x} \\
\dot{y}
\end{array}\right]=\left[\begin{array}{c}
x(3-x)-2 x y \\
y(2-y)-x y
\end{array}\right]
$$

using a perturbed form of Euler's method defined in equation (3) to find trajectories beginning at various points in the first quadrant. The 'stability' of a point, that is to say the probability that a perturbed trajectory beginning at that point will approach the same fixed point as an unpertrubed trajectory, is qualitatively displayed for points in a subset of the first quadrant in Figure 3.

Discretizing the plane and examining the variation of the average stability as a function of distance from the fixed point $(0,2)$ for a range of perturbation magnitudes results in the graph shown in Figure 4. The similarity in shape between the three graphs and the increasing vertical shift suggests that the stability distribution about $(0,2)$ is a sublinear function of $k$, the perturbation magnitude. This gives us a sense of how to measure the sort 
of instabilities exhibited in this dynamical system when there is random uncertainty in the flow.

\subsection{Further Work}

Another way of understanding the stability of a basin of attraction would be to study the impact of manipulating the constants in system (1) on the stability in terms of the linearized eigenvalues at the fixed points. This may be limited only to small radii because of the local nature of the linearization.

The metric used above does not take the geometry of the basins of attraction into account. A more refined metric might, rather than simply looking at points a distance $R$ from the fixed point to study stability fall-off, take the geometry of the basin into account. The horizontal axis on the equivalent of Figure 4 would be some function describing appropriate contour lines of the basin.

The apparently sublinear relationship between $k$ and the distribution of stability values may be a result of the fact that we choose perturbations in equation (3) uniformly in the range $[-k, k]$. A simple extension would be to change the nature of the random variable from uniform to some other distribution. A Gaussian distribution could be of particular interest since it describes the uncertainty in physical systems well. Fully describing this case would provide quantitative insight into dynamical systems with random variations in the flow lines due to a changing environment or to uncertainties in measurement. 


\section{References}

[1] Matplotlib. http://matplotlib.sourceforge.net/, 2008.

[2] Random Module. http://docs.python.org/lib/module-random.html, 2008.

[3] S.H. Strogatz. Nonlinear Dynamics and Chaos: With Applications to Physics, Biology, Chemistry, and Engineering. Perseus Books, 1994.

[4] VPython. http://vpython.org/index.html, 2008. 